\title{
Die Monoaminosäuren des «Edestins» aus Sonnenblumensamen und dessen Verhalten gegen Pankreassaft.
}

\author{
Von
}

Emil Abderhalden und Béla Reinbold.

(I. Chemisches Institut der Universität Berlin).

(Der Redaktion zugegangen am 21. März 1905.)

Die einzelnen Aminosäuren resp. deren Gruppierung im Eiweißmolekül zeigen dem Pankreasferment gegenüber ein ganz verschiedenes Verhalten. Während z. B. Tyrosin schon nach kurzer Dauer der Einwirkung von Pankreassaft auf Eiweiß abgeschieden wird, läßt sich auch nach Wochen in der Verdauungsflüssigkeit kein $\alpha$-Prolin und kein Phenylalanin nachweisen. ${ }^{1}$ Es schien uns nun von Interesse, zu verfolgen, ob auch zwischen den übrigen vom Pankreasferment abgeschiedenen Aminosäuren quantitative Unterschiede in der Schnelligkeit ihrer Abscheidung bestehen. Um dies festzustellen, arbeiteten wir nach folgendem Plane. Eiweiß von bekannter Zusammensetzung wurde mit aktiviertem Pankreassaft ${ }^{2}$ ) verdaut, und nach verschiedenen Zeitabschnitten bestimmte Mengen des Verdauungsgemisches der Dialyse unterworfen. Das Dialysat wurde hierauf mit Phosphorwolframsäure gefällt, um die komplizierteren Produkte zur Abscheidung zu bringen, und im Filtrat des Niederschlages die Monoaminosäuren bestimmt. Vorläufig haben wir den Versuch für das Tyrosin durchgeführt. Es ergab sich, daß dasselbe ziemlich rasch und vollständig abgespalten wird. Ein

1) E.Fischer u.Emil Abderhalden, Diese Zeitschrift, Bd.XXXIX, S. 81, und Bd. XL, S. 215, 1903.

2) Wir verdanken diesen Pankreassaft der Freundlickeit von Herrn Prof. Pawlow in St. Petersburg und sprechen ihm für dessen Überlassung auch an dieser Stelle unseren verbindlichsten Dank aus. 
kleiner Teil kommt in komplizierteren, dialysierbaren Verbindungen zur Abscheidung. Im nicht dialysierbaren Teil ließen sich auch nach erfolgter totaler Hydrolyse mit Säure nur geringe Spuren von Tyrosin nachweisen. Sehr rasch werden auch größere Mengen von Glutaminsäure in Freiheit gesetzt. Während der nicht dialysierbare Rest mit der Dauer der Verdauung sich stetig verringerte, zeigten die durch Phosphorwolframsäure fällbaren Produkte in ihrer Menge eine nur geringe Abnahme. Wir beabsichtigen, diese Versuche in größerem Umfange fortzusetzen und das Verhalten weiterer Aminosäuren zu verfolgen.

$\mathrm{Zu}$ den vorliegenden Versuchen wurde aus Sonnenblumensamen dargestelltes «Edestin» verwendet. Da seine Zusammensetzung noch unbekannt war, so haben wir die Monoaminosäuren bestimmt. Das untersuchte Edestin gleicht den übrigen untersuchten Edestinen in seiner Zusammensetzung. Es wurden folgende Spaltprodukte erhalten: Glykokoll, Alanin, Aminovaleriansäure, $\alpha$-Prolin, Leucin, Glutaminsäure, Asparaginsäure, Phenylalanin, Tyrosin und Serin.

\section{Experimenteller Teil.}

\section{Hydrolyse des Edestins aus Sonnenblumensamen.}

$1000 \mathrm{~g}$ Edestin aus Sonnenblumensamen mit einem Aschengehalt von $0,3 \%$ und einem Wassergehalt von $12,0 \%$ wurden mit der dreifachen Menge rauchender Salzsäure (vom spez. Gew. 1,19) 6 Stunden am Rückflußkühler gekocht. Beim Einengen der Hydrolysenflüssigkeit auf etwa die Hälfte erfolgte beim Stehen auf Eis reichliche Abscheidung von Glutaminsäurechlorhydrat. Die Kristallmasse wurde abgesaugt, mit konzentrierter Salzsäure ausgewaschen, dann in heißem Wasser gelöst, die Flüssigkeit durch Kochen mit Tierkohle entfärbt und nun in das fast ganz farblose Filtrat bis zur Sättigung gasförmige Salzsäure eingeleitet. Beim Stehen auf Eis erstarrte bald die ganze Masse kristallinisch. Die Ausbeute an reinem Glutaminsäurechlorhydrat betrug $100 \mathrm{~g}$. Zur Darstellung der freien Säure wurde aus einer Probe die Salzsäure durch Kochen mit gelbem Bleioxyd entfernt, das gelöste Blei mit Schwefelwasserstoff 
gefällt, und das Filtrat vom Bleisulfit bis zur Kristallisation eingeengt.

0,1725 g Substanz gaben $0,2574 \mathrm{~g} \mathrm{CO}_{2}$ und $0,0948 \mathrm{~g} \mathrm{H}_{2} \mathrm{O}$

Berechnet für $\mathrm{C}_{5} \mathrm{H}_{9} \mathrm{NO}_{4}$ : Gefunden:

$40,82 \% \mathrm{C}$ und $6,12 \% \mathrm{H} . \quad 40,70 \% \mathrm{C}$ und $6,06 \% \mathrm{H}$.

Die Mutterlauge des Glutaminsäurechlorhydrats wurde in der bekannten Weise eingedampft, mit Alkohol und trockener gasförmiger Salzsäure verestert, und die Ester in Freiheit gesetzt. Bei der Destillation der Ester wurden folgende Fraktionen erhalten:

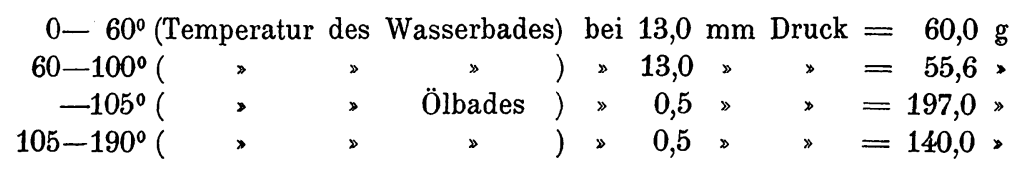

Im Destillationskolben verblieb ein dunkelbraun gefärbter Rückstand (110 g).

\section{Fraktion I.}

Die erste Fraktion bestand zum größten Teil aus Alkohol. Sie wurde mit dem zweifachen Volumen konzentrierter Salzsäure übergossen, auf dem Wasserbad vollständig zur Trockene verdampft, der Rückstand mit der gleichen Gewichtsmenge absoluten Alkohols versetzt und bis zur Sättigung trockene, gasförmige Salzsäure eingeleitet. Nach dem Impfen eines Kriställchens von Glykokollesterchlorhydrat und längerem Stehen in einer Kältemischung erstarrte die ganze Flüssigkeit kristallinisch. Auf freies Glykokoll umgerechnet ergab sich eine Ausbeute von $21,5 \mathrm{~g}$ Glykokoll.
$0,1904 \mathrm{~g}$ Substanz ergaben $0,2412 \mathrm{~g} \mathrm{CO}_{8}$ und $0,1245 \mathrm{~g} \mathrm{H}_{8} \mathrm{O}$ Berechnet für $\mathrm{C}_{4} \mathrm{H}_{10} \mathrm{NO}_{2} \mathrm{Cl}$ : Gefunden: $34,4 \% \mathrm{C}$ und $7,17 \% \mathrm{H}$. $\quad 34,55 \% \mathrm{C}$ und $7,27 \% \mathrm{H}$. Schmelzpunkt $144^{\circ}$ (korr.).

Die Mutterlauge des salzsauren Glykokollesters wurde durch Kochen mit Bleioxyd von der Salzsäure befreit, das gelöste Blei mit Schwefelwasserstoff gefällt, und das Filtrat vom Bleisulfit bis zur Kristallisation eingeengt. Es verblieben 2,5 g einer bei $293^{\circ}$ (unkorr.) schmelzenden Substanz, deren Eigenschaften auf Alanin schließen ließen. 
Fraktion II.

Sie wurde durch 8 stündiges Kochen mit der fünffachen Menge Wasser verseift, und hierauf unter vermindertem Druck vollständig zur Trockene verdampft. Der Rückstand wurde zur Entfernung des $\alpha$-Prolins mit absolutem Alkohol ausgekocht, und der alkoholische Auszug mit dem aus der nächsten Fraktion erhaltenen vereinigt. Der Rückstand selbst bestand zum größten Teil aus Alanin $(22,0 \mathrm{~g})$, ferner aus Aminovaleriansäure ( $5 \mathrm{~g})$ und aus Leucin $(3,5 \mathrm{~g})$. Die Trennung dieser Aminosäuren erfolgte zunächst durch einfache Kristallisation. Es gelang so leicht, das Leucin vollständig abzutrennen. Schwieriger gestaltet sich die Trennung von Alanin und Aminovaleriansäure.

Mit Hilfe der Kupfersalze gelang es, durch wiederholte fraktionierte Kristallisation Produkte zu erhalten, deren Fraktionen einesteils Analysenzahlen gaben, welche denen des Alanins sehr nahe standen, und andererseits Werte, welche ganz gut auf aminovaleriansaures Kupfer stimmten. Um völlig reine Aminovaleriansäure zu gewinnen, wurde ein Teil der Kristallmasse in der bekannten Weise ${ }^{1}$ ) durch Erhitzen mit Baryt auf $150^{\circ}$ im Autoklaven racemisiert. Nach dem quantitativen Ausfällen des Baryts mit Schwefelsäure gelang es nun ganz leicht, durch fraktionierte Kristallisation reine Aminovaleriansäure darzustellen :

$0,1502 \mathrm{~g}$ Substanz gaben $0,2811 \mathrm{~g} \mathrm{CO}_{2}$ und $0,1298 \mathrm{~g} \mathrm{H}_{2} \mathrm{O}$

Berechnet für $\mathrm{C}_{5} \mathrm{H}_{11} \mathrm{NO}_{2}$ : Gefunden:

$51,28 \% \mathrm{C}$ und $9,40 \% \mathrm{H}$. $51,04 \% \mathrm{C}$ und $9,6 \% \mathrm{H}$.

Das aus der Mutterlauge der Aminovaleriansäure mit Alkohol gefällte Alanin gab folgende Zahlen:

$0,1600 \mathrm{~g}$ Substanz gaben $0,2382 \mathrm{~g} \mathrm{CO}_{2}$ und $0,1144 \mathrm{~g} \mathrm{H}_{2} \mathrm{O}$

Berechnet für $\mathrm{C}_{3} \mathrm{H}_{7} \mathrm{NO}_{2}$ : Gefunden:

$40,45 \% \mathrm{C}$ und $7,87 \% \mathrm{H} . \quad 40,6 \% \mathrm{C}$ und $7,94 \% \mathrm{H}$.

Schmelzpunkt $293^{\circ}$ (unkorr.).

Das isolierte Leucin hatte folgende Zusammensetzung:

$0,2379 \mathrm{~g}$ Substanz gaben $0,4792 \mathrm{~g} \mathrm{CO}_{2}$ und $0,2117 \mathrm{~g} \mathrm{H}_{2} \mathrm{O}$

Berechnet für $\mathrm{G}_{6} \mathrm{H}_{13} \mathrm{NO}_{2}$ :

Gefunden :

$54,96 \% \mathrm{C}$ und $9,92 \% \mathrm{H}$. $54,93 \% \mathrm{C}$ und $9,89 \% \mathrm{H}$.

1) Emil Fischer und Emil Abderhalden, Ber. d. Deutschen chem. Gesellsch., Bd. XXXVII, S. 3071, 1904. 


\section{Fraktion III.}

Die Verarbeitung dieser Fraktion erfolgte in derselben Weise, wie diejenige der Fraktion II. Auch hier wurde das mit Wasser verseifte, eingedampfte Aminosäuregemisch zunächst mit absolutem Alkohol extrahiert, und hierauf aus Wasser fraktioniert kristallisiert. Der weitaus größte Teil der Fraktion bestand aus Leucin (110 g).

0,1764 g Substanz gaben 0,3541 g CO $\mathrm{C}_{2}$ und $0,1573 \mathrm{~g} \mathrm{H}_{2} \mathrm{O}$

Berechnet für $\mathrm{C}_{6} \mathrm{H}_{13} \mathrm{NO}_{2}$ :

Gefunden :

$54,96 \% \mathrm{C}$ und $9,92 \% \mathrm{H}$. $54,75 \% \mathrm{C}$ und $9,91 \% \mathrm{H}$.

Aus der Mutterlauge des Leucins wurden noch $15,5 \mathrm{~g}$ Alanin isoliert. Aminovaleriansäure war in geringer Menge neben Alanin unzweifelhaft vorhanden. Sie konnte jedoch nicht völlig gereinigt werden.

$0,1822 \mathrm{~g}$ Substanz gaben $0,3536 \mathrm{~g} \mathrm{CO}_{2}$ und $0,1597 \mathrm{~g} \mathrm{H}_{2} \mathrm{O}$

Berechnet für $\mathrm{C}_{5} \mathrm{H}_{11} \mathrm{NO}_{2}$ :

$51,28 \% \mathrm{C}$ und $9,40 \% \mathrm{H}$.

Gefunden:

Die vereinigten alkoholischen Auszüge wurden zur Isolierung des $\alpha$-Prolins zunächst unter vermindertem Druck völlig zur Trockene eingedampft. Der Rückstand wog 25,2 g. Zur Trennung des aktiven und racemischen Prolins wurde die ganze Masse mit überschüssigem Kupferoxyd gekocht, vom überschüssigen Kupferoxyd abfiltriert, und das tiefblaue Filtrat zur Trockene verdampft, und nun durch Auskochen mit absolutem Alkohol das aktive Prolinkupfer zur Lösung gebracht. Der in Alkohol unlösliche Teil der Kupfersalze wurde in Wasser gelöst und durch Einengen zur Kristallisation gebracht.

Das bei $120^{\circ}$ getrocknete Kupfersalz hatte folgende $\mathrm{Zu}$ sammensetzung:

$0,1875 \mathrm{~g}$ Substanz gaben $0,2824 \mathrm{~g} \mathrm{CO}_{2}$ und $0,0961 \% \mathrm{H}_{2} \mathrm{O}$

Berechnet für $\mathrm{C}_{10} \mathrm{H}_{18} \mathrm{O}_{4} \mathrm{~N}_{2} \mathrm{Cu}$ :

$41,16 \% \mathrm{C}$ und $5,49 \% \mathrm{H} . \quad 41,07 \% \mathrm{C}$ und $5,69 \% \mathrm{H}$.

Gefunden:

Fraktion IV.

Die aus Asparaginsäure, Glutaminsäure, Phenylalanin und Serin bestehende Fraktion wurde in der bekannten Weise zunächst durch Abscheidung des Phenylalaninesters mit Äther, 
Verseifung der nicht in Äther löslichen Ester mit Baryt und Trennung der noch vorhandenen Glutaminsäure als Hydrochlorat verarbeitet. Die Mutterlauge des Glutaminsäurechlorhydrates wurde durch Kochen mit Bleioxyd von der Salzsäure befreit, und dann durch Kristallisation die Asparaginsäure von dem Serin, das sich beim Kochen der wässerigen Lösung durch das Auftreten eines an Fleischextrakt erinnernden Geruches bemerkbar machte, zu trennen versucht. Die Trennung war eine sehr mühsame und verlustreiche. Es wurden schließlich $1,7 \mathrm{~g}$ reines Serin gewonnen.

Die Ausbeute an Phenylalanin betrug 35,6 g, an Glutamin-' säure $18,9 \mathrm{~g}$ und an Asparaginsäure 28,5 g.

Die Analyse des Phenylalanins ergab:

0,1644 g Substanz gaben $0,3928 \mathrm{~g} \mathrm{CO}_{\mathrm{z}}$ und $0,0966 \mathrm{~g} \mathrm{H}_{\mathbf{8}} \mathrm{O}$

Berechnet für $\mathrm{C}_{9} \mathrm{H}_{11} \mathrm{NO}_{2}$ :

Gefunden :

$65,45 \% \mathrm{C}$ und $6,66 \% \mathrm{H}$. $65,16 \% \mathrm{C}$ und $6,53 \% \mathrm{H}$.

Die Glutaminsäure gab folgende Werte:

$0,1802 \mathrm{~g}$ Substanz gaben $0,2676 \mathrm{~g} \mathrm{CO}_{\mathbf{8}}$ und $0,1020 \mathrm{~g} \mathrm{H}_{2} \mathrm{O}$

Berechnet für $\mathrm{C}_{6} \mathrm{H}_{8} \mathrm{NO}_{4}$ :

Gefunden:

$40,81 \% \mathrm{C}$ und $6,12 \% \mathrm{H}$. $\quad 41,0 \% \mathrm{C}$ und $6,29 \% \mathrm{H}$.

Analyse der Asparaginsäure:

0,2094 g Substanz gaben 0,2777 $\mathrm{g} \mathrm{CO}_{2}$ und 0,0992 $\mathrm{g} \mathrm{H}_{2} \mathrm{O}$

Berechnet für $\mathrm{C}_{4} \mathrm{H}_{7} \mathrm{NO}_{4}$ : Gefunden:

$36,09 \% \mathrm{G}$ und $5,26 \% \mathrm{H}$. $36,17 \% \mathrm{C}$ und $5,27 \% \mathrm{H}$.

Der bei der Destillation der Ester im Fraktionierkolben verbleibende Rückstand wurde zunächst mit viel Essigäther extrahiert. Nach dem Abdampfen des Essigäthers verblieben 1,2 g Rückstand, der alle Eigenschaften des Leucinimids zeigt (unlöslich in verdünnter Salzsäure). Der in Essigäther unlösliche Rest wurde mit überschüssigem Baryt 10 Stunden gekocht, dann der Baryt quantitativ mit Schwefelsäure entfernt. Beim Einengen des Filtrates vom Baryumsulfat schied sich zunächst Glutaminsäure aus $(10 \mathrm{~g})$. Die Mutterlauge der Glutaminsäure bildete beim weiteren Einengen einen sirupösen Brei, der ganz offenbar noch Glutaminsäure einschloß. Zu deren völliger Entfernung wurde die ganze Masse zur Trockene verdampft, in konzentrierter Salzsäure aufgenommen und noch gasförmige Salzsäure bis zur 
völligen Sättigung eingeleitet. Beim Stehen auf Eis schieden sich noch $6 \mathrm{~g}$ Glutaminsäurechlorhydrat aus. Aus dessen Mutterlauge wurde die Salzsäure durch Bleioxyd entfernt, das gelöste Blei mit Schwefelwasserstoff gefällt, und das Filtrat vom Bleisulfit eingeengt. Die Flüssigkeit roch stark nach Fleischextrakt. Es gelang nicht, kristallinische, einheitliche Produkte zu erhalten. Ein Teil war in absolutem Alkohol löslich und fiel mit Äther als weißer, flockiger Niederschlag, der an der Luft sich rasch bräunte und schmierig wurde. Der Versuch, ein kristallisiertes Kupfersalz zu gewinnen, schlug gleichfalls fehl. Auch aus dem in Alkohol unlöslichen Teil konnten keine sicher einheitlichen Produkte gewonnen werden. Es gelang zwar, kristallisierte Kupfersalze darzustellen. Ihre Menge war jedoch einesteils gering und anderenteils lag nach dem Aussehen der Kristallformen keine Garantie für Einheitlichkeit vor. Die Untersuchung muß an einem größeren Materiale wiederholt werden.

\section{Bestimmung des Tyrosins.}

Sie erfolgte in der wiederholt beschriebenen Weise aus $500 \mathrm{~g}$ Edestin. Ausbeute an reinem Tyrosin 8,9 g.

Auf $100 \mathrm{~g}$ asche- und wasserfreies Eiweiß berechnet ergeben sich folgende Mengenverhältnisse an Aminosäuren.

$\begin{array}{lr}\text { Glykokoll } & 2,5 \% \\ \text { Alanin } & 4,5 \% \\ \text { Aminovaleriansäure } & 0,6 \% \\ \text { a-Prolin } & 2,8 \% \\ \text { Leucin } & 12,9 \% \\ \text { Glutaminsäure } & 13,0 \% \\ \text { Asparaginsäure } & 3,2 \% \\ \text { Phenylalanin } & 4,0 \% \\ \text { Tyrosin } & 2,0 \% \\ \text { Serin } & 0,2 \%\end{array}$

II. Verdauungsversuch.

$500 \mathrm{~g}$ Edestin aus Sonnenblumensamen, welches 12,0\% Wasser und 0,3\% Aschenbestandteile enthielt und demnach $448 \mathrm{~g}$ asche- und wasserfreiem Eiweißkörper entsprach, wurden am 22. Dez. 1904 in 51 destilliertem Wasser suspendiert, mit 
hinreichender Menge Toluol und $80 \mathrm{ccm}$ durch Zusatz von Darmsaft aktiviertem Pankreassaft versetzt, unter häufigem Umschütteln im Brutschrank bei Körpertemperatur aufbewahrt. Im Laufe der ersten Woche ging die ganze Edestinmenge bis auf einen verhältnismäßig geringen, lockeren, grauen Bodensatz in Lösung. Dieser Bodensatz zeigte auch später keine Veränderung.

Von diesem Verdauungsgemisch wurden nach 14 Tagen (5. Jan.) und nach 21 Tagen (12. Jan.) je ein Drittel entnommen und nach 34 Tagen (25. Jan.) auch das letzte Drittel in Verarbeitung gezogen. Diese Portionen, welche nun mit A, B und C bezeichnet werden sollen, enthielten also die Verdauungsprodukte von je $149 \mathrm{~g}$ aschefreiem, trockenem Edestin und wurden in gleicher Weise verarbeitet, indem sie zunächst der Dialyse gegen destilliertes Wasser unterworfen wurden. Die Dialyse wurde in Pergamentschläuchen ausgeführt und unter täglichem - gegen Ende der Dialyse zweitägigem - Wechseln des Dialysierwassers so lange fortgeführt, bis eine Probe des Dialysats beim Eindampfen keinen erheblichen Rest zurückließ. Die Dialyse dauerte bei A vom 6. bis 13. Jan., bei B vom 17. bis 25. Jan., bei $\mathrm{G}$ vom 26. Jan. bis 8. Febr.

Das hellgelbe Dialysat, welches bei allen drei Portionen Biuret- und Millonsche Reaktion und reichliche Fällung mit Phosphorwolframsäure gab, wurde unter vermindertem Druck bei einer $40^{\circ}$ nicht übersteigenden Temperatur bis auf ein kleines Volumen eingeengt. Dabei schied sich aus A und B ein weißer Niederschlag aus, welcher im ersten Falle 3,1 g, im zweiten 2,3 $\mathrm{g}$ wog und sich bei der weiteren Prüfung als Tyrosin erwies. Von Portion $\mathrm{C}$ schied sich nur ein ganz geringer $(0,1 \mathrm{~g})$ grauer Niederschlag aus, welcher mit Millons Reagens starke Rotfärbung zeigte. Offenbar wurde in B und C ein Teil des Tyrosins durch irgend welche Ursache in Lösung gehalten, wie die starke Millonsche Reaktion der Mutterlauge des ausgeschiedenen Tyrosins bewies. Auch ist an eine Umwandlung des Tyrosins in Oxyphenyläthylamin zu denken. Es ist uns jedoch bei der Einwirkung von reinem Pankreassaft auf Eiweiß und auf Tyrosin nicht gelungen, die genannte Base zu isolieren. 
Die filtrierten Flüssigkeiten wurden jetzt mit destilliertem Wasser bis auf $6 \mathrm{l}$ aufgefüllt und mit überschüssiger Phosphorwolframsäure gefällt.

Die sehr reichliche Fällung wurde nach dem Absaugen in Wasser noch einmal suspendiert, aufgekocht, abgekühlt, der Niederschlag abgenutscht, zur gänzlichen Entfernung der Mutterlauge noch mit der hydraulischen Presse abgepreßt und dann mit überschüssigem Baryt zerlegt. Der Niederschlag wurde abgesaugt, und im Filtrat der überschüssige Baryt mit Schwefelsäure quantitativ entfernt. Das Filtrat vom Baryumsulfat wurde im Vacuum zur Trockene verdampft. Der Rückstand gab noch deutliche Biuretreaktion und zeigte mit Millons Reagens eine schwache, aber deutliche Rotfärbung. Der gesamte Rückstand wurde durch Kochen mit der dreifachen Menge konzentrierter Salzsäure hydrolysiert und durch Veresterung qualitativ nachgewiesen, daß alle im Edestin nachgewiesenen Monoaminosäuren vorhanden waren. Bei sämtlichen übrigen dieser Partie entsprechenden Produkten wurde in ganz gleicher Weise verfahren und in allen Fällen Produkte gewonnen, welche noch alle Monoaminosäuren (mit Ausnahme von Tyrosin und höchst wahrscheinlich auch von Tryptophan) in gebundener Form enthielten. Die Menge dieser Produkte nahm mit der Dauer der Verdauung etwas, aber nicht bedeutend ab.

Das von den durch Phosphorwolframsäure fällbaren Substanzen befreite Dialysat wurde nun zur Entfernung der überschüssigen Phosphorwolframsäure mit Baryt gefällt, filtriert, vom Baryt durch Schwefelsäure quantitativ befreit, und das Filtrat vom Baryumsulfat unter vermindertem Druck bei niedriger Temperatur eingeengt. Diese Rückstände enthielten alle viel Glutaminsäure. Sie wogen bei A $44,3 \mathrm{~g}$, bei B $43,4 \mathrm{~g}$, bei C $50,4 \mathrm{~g}$. Sie gaben bei den zwei ersten Portionen eine schwache, bei der letzten Portion eine sehr erhebliche Millonsche Reaktion. In dieser letzteren Portion war das Tyrosin aus unbekannten Gründen nicht wie bei den übrigen direkt beim Einengen zur $\mathrm{Ab}-$ scheidung gelangt, sondern im Sirup gelöst geblieben. Es konnte aus diesem durch Auskochen des möglichst scharf getrockneten Rückstandes mit Eisessig rein gewonnen werden. 
Der nicht dialysierte Rest des Verdauungsgemisches resp. der einzelnen Portionen wurde auf dem Wasserbade eingedampft. Der dunkelbraune Rückstand wog bei A $30 \mathrm{~g}$, bei B $26 \mathrm{~g}$ und bei C $15 \mathrm{~g}$. Er war in Wasser nur zum Teil löslich; der wasserunlösliche Teil löste sich mit Leichtigkeit in verdünnten Alkalien, fiel aber beim Neutralisieren wieder flockig aus.

Um festzustellen, ob in diesen Rückständen noch Tyrosin in freier oder gebundener Form enthalten sei, wurden sie zunächst mit Wasser ausgekocht, und die wässerige Lösung auf dem Wasserbade zur Trockene verdampft und nun mit Millons Reagens auf Tyrosin geprüft. Die Reaktion fiel bei der ersten Portion positiv aus, sie war aber äußerst schwach. Die beiden übrigen Partien enthielten kein Tyrosin. Nun wurden diese wasserlöslichen Teile und die unlöslichen vereinigt und durch 12stündiges Kochen mit 25\% \%iger Schwefelsäure gespalten. Nach quantitativer Ausfällung der Schwefelsäure mit Baryt wurde das Filtrat zur Trockene verdampft und der Rückstand auf Tyrosin untersucht. $\mathrm{Zu}$ A und B wurde eine äußerst schwache Millonsche Reaktion erhalten, $C$ enthielt kein Tyrosin in nachweisbarer Menge. 